\title{
Neuro-Nimbuzz-An Epileptic Seizure Prediction and Prevention System Using Cloud Deployment and Real-Time Analysis
}

\author{
Niyati Parameswaran, Bharathi Muthu, and Sundaram Swaminathan
}

\begin{abstract}
This paper presents Neuro-Nimbuzz- An Epileptic Seizure Predication System using Cloud Deployment and Real-Time Analysis. Neuro-Nimbuzz is intended to allow an epileptic seizure prediction system to achieve true predictive power in terms of statistical computation, analytical performance estimates and influence of real-time constraints. Intelligence incorporated to Neuro-Nimbuzz's design makes it capable of generating preferential therapies and treatments for epileptic patients by utilization of customizable dynamic updates. The model proposed focuses on reducing the bridge between a Client Application and a Cloud Environment so as to render seamless operations. Neuro-Nimbuzz's system model is closely integrated and its concept has the potential to be extended into several other customizable applications.
\end{abstract}

Index Terms-Classifier, cloud deployment, EEG, ensemble, feature extraction, real-time constraints, recommender system, seizure prediction and seizure prevention.

\section{ACRONYMS}

CBESM: Cloud Based Epileptic Seizure Prediction and Prevention Model.

SVM: Support Vector Machine

CNN: Convolutional Neural Network

RNS: Responsive Neurostimulation

UML: Unified Modeling Language.

\section{INTRODUCTION}

This paper proposes an on-line framework which thoroughly analyzes a patient's EEG samples, extracts relevant information from these samples and utilizes this information to predict the occurrence of a seizure while also providing intuition on the best possible way to suppress the seizure once its onset has been detected. The Model has been facilitated with the capacity to retrain its data by implementing Real-Time Constraints and dynamic computation methodologies to augment both the trained and cross validated datasets by gradually enlarging the sample data over time.

Fundamental concepts that are crucial for epileptic seizure prediction studies such as the degree of variation and statistical validation of prediction methodologies have been taken into consideration. Furthermore, the Framework has been incorporated with intelligence which allows it to update, review and improve its performance efficiency on the tr

Manuscript received November 7, 2012; revised January 28, 2013.

The authors are with Birla Institute of Technology \& Science Pilani, Dubai Campus, DIAC, Dubai, UAE (e-mail: niyati.15@ gmail.com, bharathi.smm@gmail.com,swami@bits-dubai.ac.ae). aining model by optimizing its post processing scheme.

The model has been designed using UML [1] as it provides a platform which permits effortless additions and deletions to the framework, allows ease of communication of the system architecture and supports modifications in the model's design to a degree as deemed fit by the developers.

The operations have been implemented using a cloud deployment system [2] in order to boost the scalability, flexibility, customizability and manageability of resources, reduce maintenance costs and the need for heavy-weight applications and also provide access to a potentially much larger bracket of people and a wider array of organizations.

\section{PROBlem Formulation}

Epilepsy is a brain disorder in which a person has repeated seizures (convulsions) over time. It is the sudden, unforeseen way in which these seizures occur that represents one of the most disabling aspects of the disease. Moreover, the intense feeling of helplessness aside from the risk of serious injury affects the patient psychologically rendering them vulnerable in a lot of circumstances [3].

A deep-seated problem with epileptic seizures is the high degree of variation and specification associated with the seizure which is subject to change for every epileptic patient [4]. Thus, the traditional training methods which involved offline training would not be very suited for the purpose as we will be impeding system performance by restricting our data to a fixed training set.

Finally, approximately 40 million people worldwide are epileptic. Epilepsy is the third most common neurological disorder following stroke and Alzheimer's disease. Present day therapy and treatments to control epilepsy include concepts and strategies that are characterized by limitations in terms of lack of specification further reinstating why a system which will be able to benefit these patients is vital.

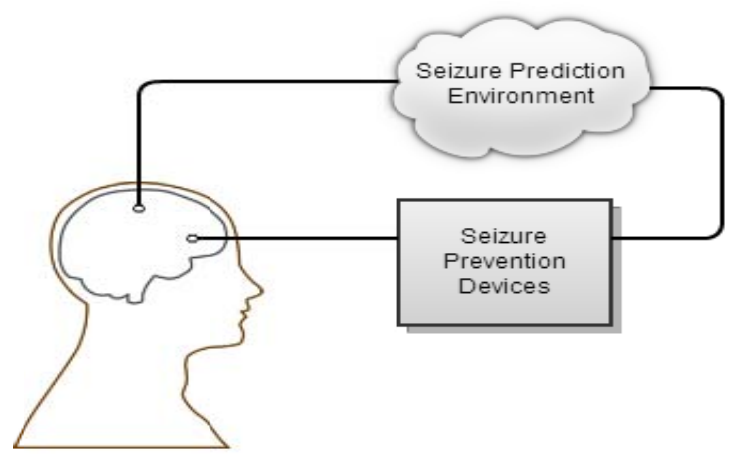

Fig. 1. Schematic block diagram for CBESM-Neuro-Nimbuzz 


\section{PROBLEM SOLUTION}

A system which has an efficient feature extraction caliber, a well-organized processor, a versatile, resourceful classifier and a competent architecture which facilitates optimization of the objective function and takes into consideration factors applicable to the patient will be able to provide adequate intuition to the medical practitioners and thus deal with the challenges that impede the seizure's prediction and its prevention.



Fig. 2. Deployment diagram of CBESM-Neuro-Nimbuzz

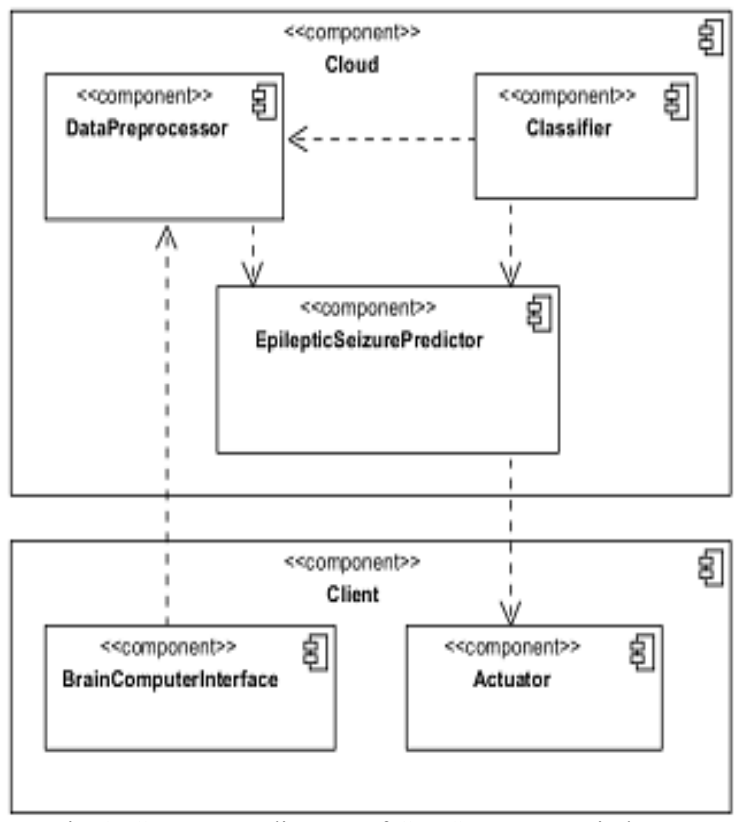

Fig. 3. Component diagram of CBESM-Neuro-Nimbuzz

The Deployment Diagram [1] as shown in Fig. 2 comprises:

1) Brain Computer Interface: A brain-computer interface [5] is a communication system that does not depend on the brain's normal output pathways of peripheral nerves and muscles. It instead conducts operations on the basis of interaction between the patient's brain and the system itself. The patient's brain is responsible for providing conclusive data on the activity particulars of the patient which is duly measured by the BCI system while the BCI system is responsible for the translation of these activity particulars into readable commands. The input is recorded by the Electrode Array in the form of an EEG Recording and a translation algorithm gets invoked which works on this data. The Data Transmitter transfers this EEG Recording from the Client side to the Cloud environment.

2) Seizure Prediction Environment: The proposed Seizure Predictor is an executable environment which will be deployed by utilization of a Cloud driven Architectural Model.

3) Preventive device: The Prevention Techniques proposed will employ an envisioning concept wherein the proposed medication will take into account the stimulation parameters which are specific to the patient, the medical history of the patient and the so far preferred treatment plan for the patient. The model also takes into consideration recommendations made by the medical practitioner.

The Component Diagram [1] as represented in Fig. 3 consists of the Client and the Cloud components. The Client contains the Brain Computer Interface-which provides the input feed to the Cloud component and the Actuator Component- which provides the patient with appropriate medication as recommended by the Cloud component while also notifying the medical practitioner by means of an alarm trigger. The Cloud Component contains the Data Preprocessor- which will scale the data so that the features of multiple EEG records will be normalized for better computation analysis and performance efficiency, the Classifier [6]- which will utilize an ensemble to create a compact state representation for epileptic patients boosting concurrency and reducing conflict relations and an Epileptic Seizure Predictor-which will suggest the optimal preferential treatment for the patient exact to the patient's history providing customizability and flexibility to the system.

The three classes which form part of the Cloud component are the Data PreProcessor (Fig. 4), (Fig. 5) the Classifier (Fig. 6) and the Epileptic Seizure Predictor (Fig. 6).

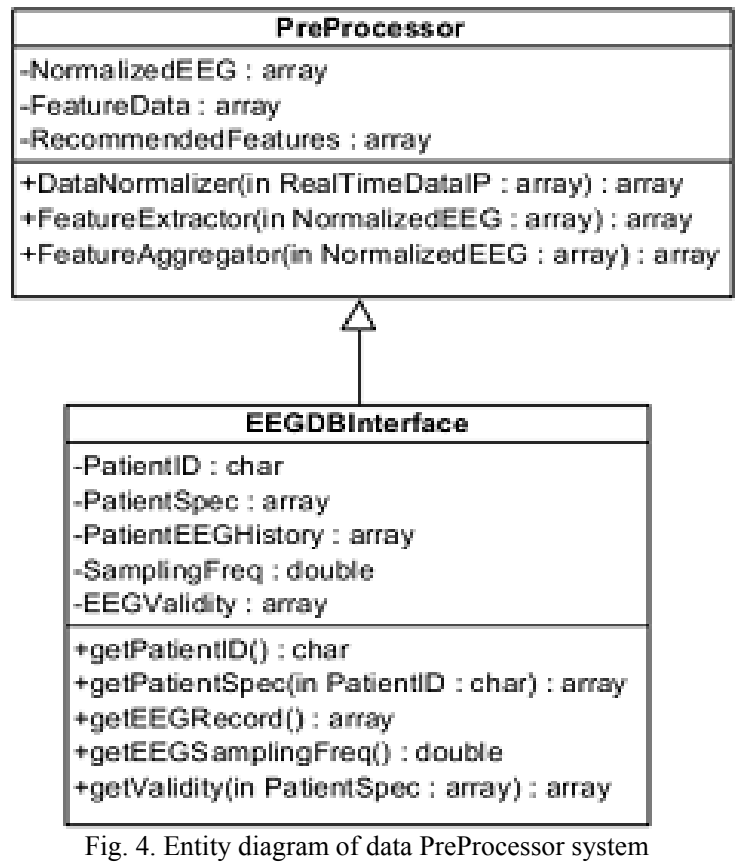

The Data PreProcessor will serve to normalize [6] the EEG 
data samples to a prefixed scale. The DataNormalizer() suitably modifies the statistical features which characterize every EEG record so that further understanding of this data is unhindered by gross influences- implying that every EEG record will be influenced to a similar degree when we revise the objective function. The FeatureExtractor() [6] will make use of pertinent information from the EEG Sample by accurately determining feature intensities and feature ratios, flagging outliers and calculating statistical confidences. The FeatureAggregator() [6] will then extort data from the EEG record while taking into consideration the patient's specifics and compile the list of features which would be most relevant to the patient's condition.

The Classifier Class consists of an Ensemble [6] and a Recommender System [7].

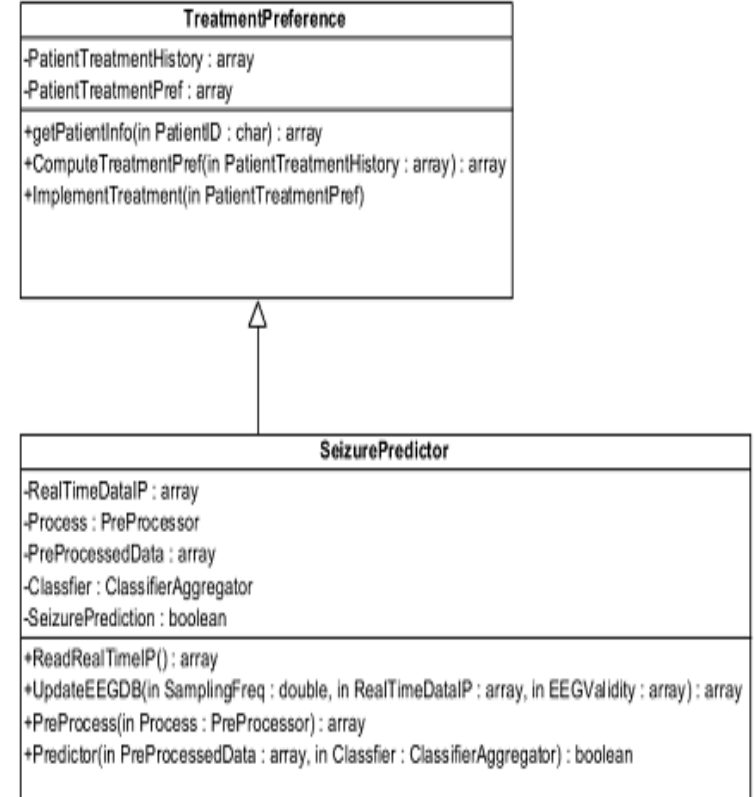

Fig. 5. Entity diagram of seizure prediction system

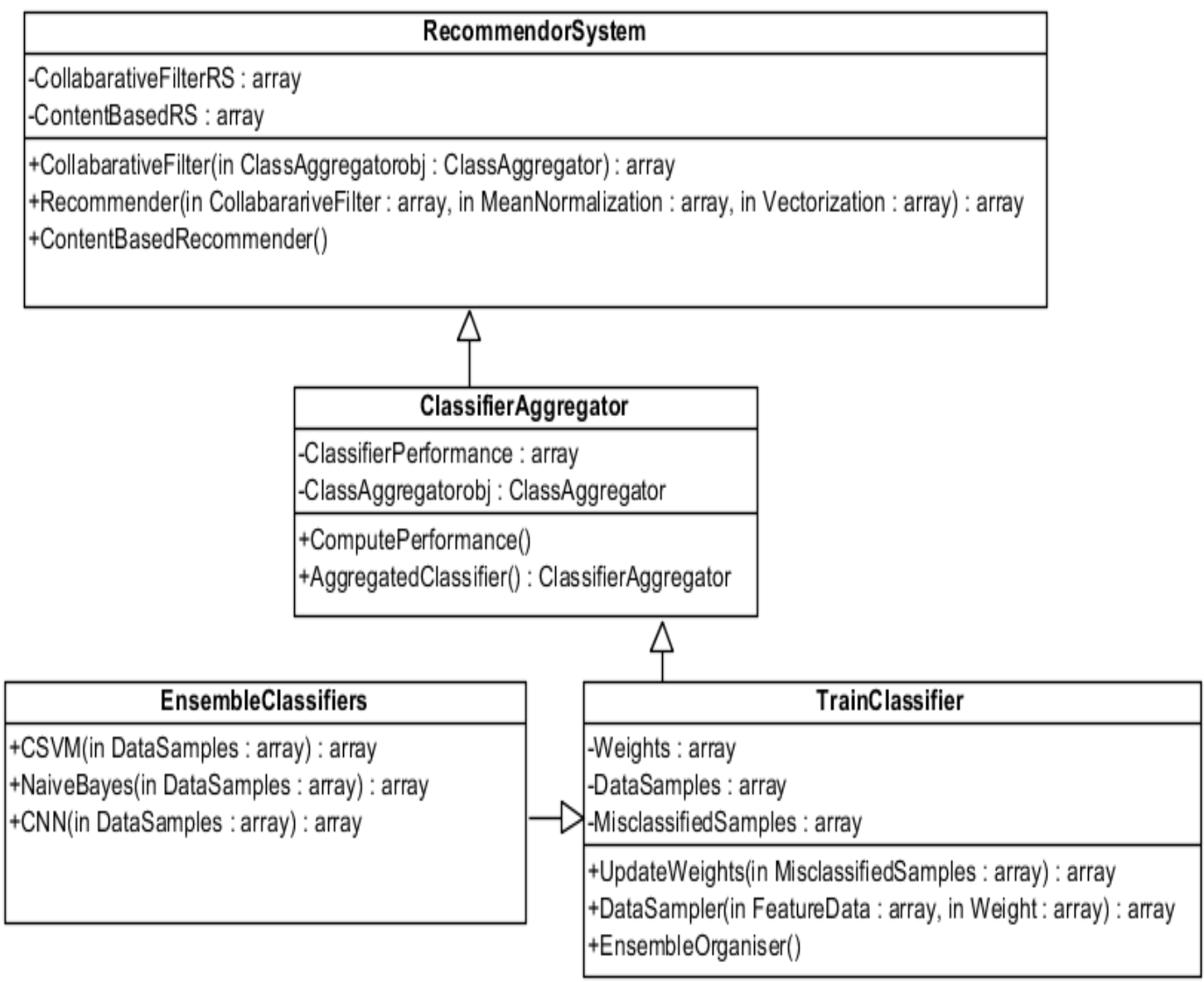

Fig. 6. Entity diagram of classifier

\section{A. Ensemble}

The Ensemble will train several models from the samples of the PatientEEGHistory dataset and combine the output predictions by aggregation of the output values for the seizure prediction problem. We will be training the Ensemble by making use of Boosting [6] which is an iterative process of adding more weights to the cases which have been classified incorrectly by the Classifier. This is followed by combining all the models generated during the process. The results obtained after Boosting are then used to produce significant model variations, improve model accuracies and reduce model variance in the Ensemble. In Neuro-Nimbuzz we propose the utilization of Support Vector Machines [6], Naïve Bayes Algorithm [6] and Convolutional Neural Networks [8] in the Ensemble.

\section{1) Support vector machines}

Assume $\mathrm{L}$ training points, where each input $x_{i}$ has $\mathrm{D}$ attributes (i.e. is of dimensionality D) and is in one of two 
classes $y_{i}=-1$ or +1 , then our training data is:

$$
\left\{x_{i}, y_{\mathrm{i}}\right\} \text { where } i=1 \ldots \mathrm{L}, y_{i} \in\{-1,1\}, x \in R^{\mathrm{D}}
$$

Here we assume the data is linearly separable and that we can draw a hyperplane [9] on graphs of $x_{1} ; x_{2}::: x_{D}$ for when $\mathrm{D}>2$. This hyperplane can be described by w. $x+b=0$ where

- $\mathrm{w}$ is nomal to the hyperplane

- $\frac{b}{\|w\|}$ is the perpendicular distance from the hyperplane to the origin.

Support Vectors are the examples closest to the separating hyperplane and the aim of Support Vector Machines (SVM) is to orientate this hyperplane in such a way as to be as far as possible from the closest members of both classes.

\section{1) The naïve bayes algorithm}

The Naïve Bayes algorithm is a classification algorithm based on Bayes rule, that assumes the attributes $X_{1} \ldots X_{n}$ are all conditionally independent [10] of one another, given $Y$. When $X$ contains $n$ attributes which are conditionally independent of one another given $\mathrm{Y}$, we have

$$
P\left(X_{1} \ldots X_{n} \mid Y\right)=\prod_{i=1}^{n} P(X i \mid Y)
$$

According to Naive Bayes algorithm, assuming $Y$ is any discrete-valued variable, and the attributes $X_{1} \ldots X_{n}$ are any discrete or real valued attributes, the expression for the probability that $Y$ will take on its $k^{\text {th }}$ possible value, according to Bayes rule, is

$$
P\left(Y=\mathrm{y}_{\mathrm{k}} \mid X_{1} \ldots X_{n}\right)=\frac{P(Y=y k) \prod_{i} P(X i \mid Y=y k)}{\sum_{j} P(Y=y j) \prod_{i} P(X i \mid Y=y j)}
$$

The most probable value of $\mathrm{Y}$ according to the Naive Bayes Classification rule gets computed to being

$$
\mathrm{Y} \leftarrow \arg \max _{y k} \frac{P(Y=y k) \prod_{i} P(X i \mid Y=y k)}{\sum_{j} P(Y=y j) \prod_{i} P(X i \mid Y=y j)}
$$

\section{2) Convolutional neural network}

CNN's are neurobiologically motivated by the findings of locally sensitive and orientation-selective nerve cells in the visual cortex of the cat. The network structure in a CNN implicitly extracts relevant features, by restricting the neural weights of one layer to a local receptive field in the previous layer. Thus, a feature map as illustrated in Fig. 7 is obtained in the second layer and consecutively in the subsequent layers. By reducing the spatial resolution [8] of the feature map, a certain degree of shift and distortion in variance is achieved. Also, the number of free parameters is significantly decreased by using the same set of weights for all features in the feature map.

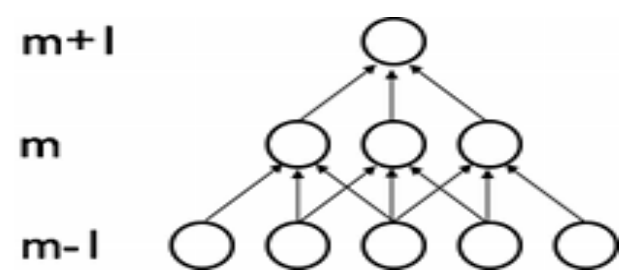

Fig. 7. Spatial local correlation in convolutional neural network.

3) Recommender system

Recommender Systems aid in improving the performance of the Machine Learning Algorithms. The system helps the algorithms of the Ensemble to identify which features while assigning the certain magnitudes of significance for every feature. Thus, the Recommender System will facilitate a comprehensive analysis of the features using case based analysis and relevant feature study. In Neuro-Nimbuzz we will use Collaborative Filtering [7] - which will collaborate the data of multiple patients who possess similar stimulation and assessment parameters and Content Based Recommender Systems [7] - which examines a specific patient's current data sample while also inspecting the previous data samples of the patient.

The Epileptic Seizure Predictor will first take in EEG data samples at real-time and normalize these data samples so that they can be worked on. The Predictor will then make use of the features of this test data to compute the likelihood of the onset of a seizure. The objective function simulated by the classifier will be utilized for this assessment. Following this the Predictor will analyze the various diagnostic tools which have worked optimally for the patient in the past and will examine case based reasoning and learning from previously operated patients with similar assessment parameters. It will go on to inspect the particular traits that dominate the preictal ('preceding the seizure'), the interictal('during the absence of a seizure'), the ictal(' during the seizure') and the postictal ('following the seizure') states of the patient [11] while continuing to monitor the dynamic transitioning of these states. It will also take into account neurobiological, cognitive and psychological factors that affect the patient. The Predictor will then update the EEG Database with the training it has gained after studying the test data and will subsequently delete the data it does not require any more accordingly. Finally, the Predictor will make available to the medical practitioner preferential treatments that would be best suited for the patient.

The Seizure Prevention will implement a Closed-Loop Control of Epilepsy. Since the aberrant brain activity that is associated with epilepsy is characterized by intermittent pattern emergence, thus a closed-loop brain feedback control which leaves other aspects of the brain functions unaffected is desirable.

The prevention mechanisms proposed are invasive techniques [5] which include placing of an imperceptible device in a section of the patient's skull. These devices can be accessed through outpatient surgery whenever the battery of the device has to be changed. These mechanisms have also been modified to provide a cloud-based interface [12] for remote patient monitoring.

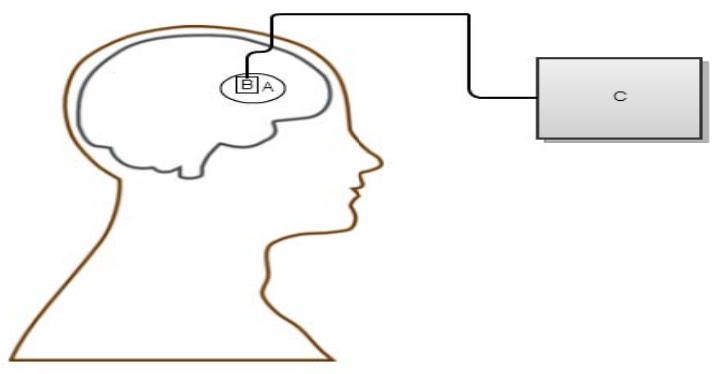

Fig. 8. Schematic diagram for seizure prevention.

\section{A. Targeted Drug Delivery [13]}

The device contains an infusion pump and catheters. The 
catheters are linked to the affected sites in the brain. The Targeted Drug Delivery technique will feed seizure-halting medications directly to the specific areas of the brain tissue whenever need be. Since $2 / 3^{\text {rd }}$ s of epileptic patients can be treated by means of anti-epileptic drugs, this method is deemed fit for a large number of epileptic patients. This procedure unlike oral medication has no systemic side effects. In fact, this medication has been able to maintain seizure control in patients over months. However, this process would require monitoring in terms of dosage and an infusion schedule by a trained medical practitioner. Its application in a linked, responsive, autonomous system could result in miscalculations and risks. (Table I)

TABLE I: DESCRIPTION OF FIG. 8 FOR CBESM-NEURO-NIMBUZZ

\begin{tabular}{|l|l|l|l|}
\hline \multicolumn{1}{|c|}{$\begin{array}{c}\text { Prevention } \\
\text { Technique }\end{array}$} & \multicolumn{1}{c|}{$A$} & \multicolumn{1}{c|}{$B$} & \multicolumn{1}{c|}{$C$} \\
\hline $\begin{array}{l}\text { Targeted Drug } \\
\text { Delivery }\end{array}$ & $\begin{array}{l}\text { Affected } \\
\text { Site(s) }\end{array}$ & Catheters & $\begin{array}{l}\text { Infusion } \\
\text { Pump }\end{array}$ \\
\hline $\begin{array}{l}\text { Deep Brain } \\
\text { Stimulation }\end{array}$ & $\begin{array}{l}\text { Affected } \\
\text { Site(s) }\end{array}$ & Electrode & $\begin{array}{l}\text { Pulse } \\
\text { Generator }\end{array}$ \\
\hline $\begin{array}{l}\text { Responsive } \\
\text { Neurostimulation }\end{array}$ & $\begin{array}{l}\text { Seizure } \\
\text { Focus }\end{array}$ & Electrode & $\begin{array}{l}\text { Neurostimul } \\
\text { ator }\end{array}$ \\
\hline Focal Cooling & $\begin{array}{l}\text { Seizure } \\
\text { Focus }\end{array}$ & $\begin{array}{l}\text { Thermo-electronic } \\
\text { cooling device }\end{array}$ & Cooling Sink \\
\hline
\end{tabular}

\section{B. Deep Brain Stimulation [14]}

The device contains leads, electrodes and a pulse generator device. The pulse generator will kindle the anterior nucleus of the thalamus, which helps control the electrical excitability of the cortex hence preventing the onset of seizures. This method makes it possible to provide a prospective treatment in case of patients where seizures have the tendency to engulf the entire brain. Moreover, this form of prevention permits the stimulation of discreet networks in very close proximity to the seizure generator. The provision for multiple implantation sites adds to the individualization of this technique. However, this practice carries the risk of hemorrhage and infection. Also, determination of the best site for electrode placement adds to the complexity of this method.

\section{Responsive Neurostimulation[15]}

The device contains leads, electrodes and a neurostimulator. The electrodes are placed in the brain at the seizure focus. The RNS will deliver electrical stimulation to interrupt this electrical activity from progressing thereby arresting the possibility of the seizure. This method provides a lot of versatility in its implementation. This process can be modified to accommodate programmable restimulations in cases of persistent seizure activity. The stimulation parameters also can be adjusted as per requirement with the option of combining this form of therapy with other physical approaches. However, the mechanical effects and the electrolysis near the electrodes could affect seizure frequency. Furthermore, determination of the best site for electrode placement is critical in this method.

\section{Focal Cooling [16]}

The device contains a cooling sink. This cooling sink is connected to the seizure focus of the patient. The Focal Cooling mechanism will respond by triggering impulses which serve to rapidly cool the stimulated region and thus halt the occurrence of the seizure. This method finds its utility especially in cases where patients have undergone or have a tendency to be susceptible to neuronal injury or excessive electrical activity in the affected sites. The potential clinical utility of therapeutic cooling or hypothermia for neurological disorders comes across as a major benefit in these situations as this cooling is neuroprotective. Like RNS, this technique too can be combined with other physical approaches. However, monitoring this system is tedious. Also, excessive focal cooling can result in tissue damage. Currently the systemic effect on other body organs is not entirely conclusive.

\section{CONCLUSION}

Epilepsy is a neurological disorder which requires a high degree of customization due to the large extent of variation in every epileptic person's condition. The design proposed takes into account this need for individualization by referencing the patient's previous medical records and the preferred proposed treatments for the patient before assembling the favored treatments in an order of preference. As the model will learn continuously from the data it works on, it becomes essential to introduce an on-line training element to the design. Dynamic computations and updates are crucial for such a system where the performance on training data needs to be optimized to fullest capacity. A Standalone application will add to the weight of the application and increase overheads in terms of data storage and costs. Thus, deploying the application onto a Cloud executable environment will help incorporate several features which re-instate its suitability for usage. The usage of the Feature Extraction, Ensemble and Recommender Systems will serve to leverage accuracy and resourcefulness to the design as the model will be retrained after every simulation with updates being made to the sample data as per requirement.

The challenges which we may face during the implementation of the Neuro-Nimbuzz are as follows. The conceptualization of the Ensemble technique may result in a lower performance on the testing data as opposed to the performance acquired on the training model. The prevention techniques proposed involve inherent risks as they are invasive and could result in an infection. Also, the prevention methodologies cannot be fully customized to suit the patient's needs due to limitations in their design. In the case of an impending seizure, monitoring the patient's health by a medical professional may still be essential as these procedures do not function to their optimal capacity as autonomous devices. Finally the connections can get dropped due to lack of network availability for a small period of time such as in cases of cloud outage and we may have to extend the design to an offline alternative to ensure seamless operations.

\section{REFERENCES}

[1] L. Craig, Applying UML and Patterns: An introduction to object-oriented analysis and design and iterative development, 3rd ed. Pearson Education, Inc., 2005, ch. 5, pp. 475-655.

[2] T. Karnwal, T. Sivakumar, and G. Aghila, "Cloud Services in Different Cloud Deployment Models. An Overview," International Journal of Computer Applications, vol. 34, no. 8, November 2011. 
[3] S. Schachter and P. Shafer. (February 2007). On the All about epilepsy and seizures. [Online]. Available: http://www.epilepsy.com/Epilepsy/main epilepsy

[4] S. Bonhage and Kühn, Unpredictability of Seizures and the Burden of Epilepsy, in Seizure Prediction in Epilepsy: From Basic Mechanisms to Clinical Applications, B. Schelter, J. Timmer and A. S. Bonhage, Wiley-VCH Verlag GmbH \& Co. K Ga A, Weinheim, Germany, 2008

[5] N. Birbaumer, W. J. Heetderks, D. J. McFarland, P. H. Peckham, G. Schalk, E. Donchin, L. A. Quatrano, and C. Robinson, "Brain-Computer Interface Technology: A Review of the First International Meeting," IEEE transactions on rehabilitation engineering, vol. 8, no. 2, June 2000.

[6] Tan, Steinbach and Kumar, Introduction to Data Mining, Addison Wesley, 2006.

[7] J. A. Konstan. (2008). On the Introduction to Recommender Systems. [Online]. Available: http://www-users.cs.umn.edu/ konstan/SIGMOD-2008-Tut.pdf

[8] D. Bouchain, "Character Recognition Using Convolutional Neural Networks," in Seminar Statistical Learning Theory, Germany, 2006, pp. 6-7

[9] C. Cortes and V. Vapnik, in Machine Learning, pp. 273-297, 1995.

[10] T. Mitchell, Machine Learning, McGraw Hill, 1997.

[11] C. Y. Chiang, "Algorithm and Architecture Design of Epilepsy Seizure Prediction System," Ph.D. dissertation, Graduate Institute of Electronics Engineering, National Taiwan University, Taipei, R.O.C., 2011.

[12] The NIST Definition of Cloud Computing, National Institute of Standards and Technology, Special Publication.

[13] A. V. Dycke, R. Raedt, K. Vonck, and P. Boon, "Local delivery strategies in epilepsy; a focus on adenosine," Seizure: European journal of Epilepsy, vol. 20, pp. 376-382, June 2011.

[14] S. Breit, J. Schulz, and A. L. Benabid, "Deep brain stimulation," Cell Tissue Res, pp. 275-288, 2004.

[15] S. P. Bingulac, "On the compatibility of adaptive controllers," in Proc. 4th Annu. Allerton Conf. Circuits and Systems Theory, New York, 1994, pp. 8-16.

[16] H. Imoto, M. Fujii, J. Uchiyama, et al., Use of a Peltier chip with a newly devised local brain-cooling system for neocortical seizures in the rat, Technical note. J Neurosurg, 2006,vol. 104, no. 1.

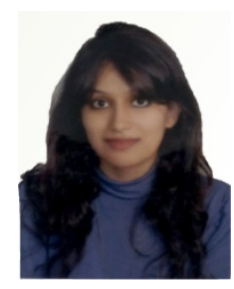

Niyati Parameswaran is pursuing her final year in Computer Science \& Engineering at Birla Institute of Technology \& Science Pilani, Dubai Campus. She intends to pursue specialization and do substantial research. She is proficient in $\mathrm{C}, \mathrm{C}++, \mathrm{JAVA}, \mathrm{VB}$, Octave, Perl and jQuery. Her fields of interest include Machine Learning, Computational Intelligence, Neural Interface Systems, Web Security, Crypt Analysis, Cloud Computing and Literature.

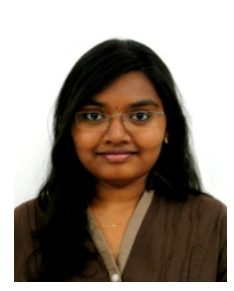

Bharathi Muthu is pursuing her final year in Computer Science \& Engineering at Birla Institute of Technology \& Science Pilani, Dubai Campus. She intends to pursue Operations Management. She is proficient in $\mathrm{C}, \mathrm{C}++$, JAVA, XML and UML. Her fields of interest include Data Mining, Cloud Computing, Digital Electronics and Computer Organizations, Artificial Intelligence and Neural Interface Systems.

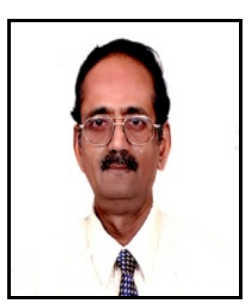

Sundaram Swaminathan is currently a professor at Birla Institute of Technology \& Science Pilani, Dubai Campus. He obtained his $\mathrm{PhD}$ (Engineering) in Semiconductor Devices from the University of Warwick in 1985. He has over 35 years of teaching and research at senior level at IIT Roorkee, University of Warwick and NTU, Singapore. His fields of specialization include Biomedical Instrumentation, Biophotonics, Biometrics, Telemedicine, Microelectronics and Nanotechnology. He has been actively involved in a number of research initiatives at a global level and has several peer-reviewed International Journals and Publications to his credit. He has been a Senior Member of IEEE since 1992. 